\title{
Detection of Illicit Substances in Fingerprints by Infrared Spectral Imaging
}

\author{
Ping Hei Ronnie Ng, Sarah Walker, Mark Tahtouh and Brian Reedy* \\ Centre for Forensic Science, University of Technology, Sydney, PO Box 123 Broadway NSW 2007 \\ Australia
}

\begin{abstract}
FTIR and Raman spectral imaging can be used to simultaneously image a latent fingerprint and detect exogenous substances deposited within it. These substances might include drugs of abuse or traces of explosives or gunshot residue. In this work, spectral searching algorithms were tested for their efficacy in finding targeted substances deposited within fingerprints. "Reverse" library searching, where a large number of possibly poor quality spectra from a spectral image are searched against a small number of high quality reference spectra, poses problems for common search algorithms as they are usually implemented. Out of a range of algorithms which included conventional Euclidean distance searching, the spectral angle mapper (SAM) and correlation algorithms gave the best results when used with second derivative image and reference spectra. All methods tested gave poorer performances with first derivative and undifferentiated spectra. In a search against a caffeine reference, the SAM and correlation methods were able to correctly rank a set of 40 confirmed but poor quality caffeine spectra at the top of a dataset which also contained 4096 spectra from an image of an uncontaminated latent fingerprint. These methods also successfully and individually detected aspirin, diazepam and caffeine that had been deposited together in another fingerprint, and they did not indicate any of these substances as a match in a search for another substance which was known not to be present. The SAM was used to successfully locate explosive components in fingerprints deposited on silicon windows. The potential of other spectral searching algorithms used in the field of remote sensing is considered, and the applicability of the methods tested in this work to other modes of spectral imaging is discussed.
\end{abstract}

\section{Keywords}

FTIR imaging, spectral library searching, fingerprints, explosives, drugs of abuse

*Corresponding author:

Brian Reedy

Centre for Forensic Science

University of Technology, Sydney,

PO Box 123

Broadway NSW 2007

Australia

brian.reedy@uts.edu.au 


\section{Introduction}

The use of vibrational spectroscopic techniques (Fourier transform infrared and Raman) has become increasingly important in forensic science, and the relatively recent development of spectral mapping and imaging instrumentation has given rise to exciting new applications in fingerprint enhancement [1-12] and trace evidence [13-16]. These two areas of forensic science intersect in studies that seek to identify trace evidence, such as particles of explosives or illicit drugs, within fingerprint deposits. Spectral or chemical imaging is generally rapid and non-destructive, and simultaneously delivers spatial information about a fingerprint (i.e. an image) that can help to identify a suspect, and chemical information that might indicate substances handled by that person. The present study examines techniques for locating compounds of interest within a latent fingerprint, using spectral image data. Among other issues, this will often involve searching for a small number of low quality target spectra among thousands of "empty" spectra, a problem that is not necessarily confined to fingerprint evidence, nor to vibrational spectroscopy.

Latent fingerprints are a complex mixture of eccrine and sebaceous materials $[17,18]$ that may be contaminated by exogenous substances of various sources. FTIR and Raman spectroscopy have been demonstrated as powerful tools for examining both the endogenous and exogenous materials found in latent fingerprints. Wilkinson et al $[19,20]$, Bartick et al $[2,4]$ and Williams et al [21] used single point FTIR microscopy to chemically characterise latent fingerprints from adults. Day et al [22] examined the Raman spectra of latent fingerprints as part of a study on the detection of drugs of abuse. Hemmila et al [23] have recently used FTIR reflectance spectra of latent prints, with partial least squares regression, to show that fingerprints could be used to gauge the age of an individual, based on the fingerprint composition. Apart from these, studies on uncontaminated latent fingerprints have tended to involve the use of FTIR imaging techniques. Bartick et al [2, 4], Tahtouh et al [5] and Crane et al [9] demonstrated the micro-reflectance FTIR imaging of latent fingerprints on a variety of surfaces, while Ricci et al developed the ATR-FTIR imaging of latent fingerprints deposited directly onto the ATR crystal [11] and latent fingerprints on a gel lifter [10]. An important method for enhancing latent fingerprints is their development by cyanoacrylate fuming, which aims to cause a white polymer to form preferentially on the ridges of a fingerprint. Tahtouh et al $[5,12]$ have demonstrated and optimised the micro-reflectance mode FTIR imaging of cyanoacrylatedeveloped fingerprints on non-porous surfaces, using spectral second derivatives to enhance ridge contrast in the images. 
More recently, interest has focused on the spectroscopic detection of exogenous particles which might be found within latent fingerprint deposits, particularly explosives and drugs of abuse. The early work in this area involved Raman microscopy on explosive-contaminated fingerprints $[1,24$, 25]. More recent Raman work has been carried out by Day et al on the detection of drugs of abuse in latent [22] and cyanoacrylate-fumed [26, 27] fingerprints. The latter articles sought to determine whether spectral or chemical interference from the cyanoacrylate could be removed or minimised. West and Went $[28,29]$ have shown that Raman spectroscopy can be used to detect exogenous material such as drugs of abuse in fingerprints that have been developed with powders and lifted with adhesive tape.

Most infrared studies of contaminants in latent fingerprints have involved spectral imaging. Crane et al [9] were able to recover and analyse trace evidence found in a fingerprint on a postcard. Meanwhile, Chan and Kazarian [16] and Ricci et al $[8,10,11]$ have demonstrated the ability of ATRFTIR imaging to detect trace materials with high sensitivity in fingerprints directly or indirectly (via gel lifters) deposited onto the ATR crystal. In the latter case, the use of a variable angle ATR accessory enabled the separation of the spectra of the surface contaminants from those of the lifter itself, because imaging could be performed at different depths of penetration into the sample. The ability to detect contaminants in lifted fingerprints is important because otherwise FTIR imaging is limited to (i) prints deposited on flat surfaces which are amenable in both size and composition to micro-reflection or ATR imaging and (ii) prints deposited by suspects in custody onto appropriately reflective slides or infrared-transparent windows. In the latter case, transmission is the preferred technique, since the spectra are less likely to be distorted (than in reflection), and multiple fingerprints can be recorded and stored for further analysis (not practical with ATR).

Grant et al also assessed the ability of infrared microspectroscopy (including imaging/mapping) to detect and identify particles of recently handled materials in latent fingerprints [7]. They trialled conventional and synchrotron infrared sources in the detection of particles of different sizes, recommending the latter for the smallest particles, despite the relative inaccessibility of synchrotron radiation to forensic scientists. They also examined the use of (single point) micro-ATR FTIR spectroscopy in detecting contaminant particles and concluded that while ATR gives higher quality spectra, it is a destructive technique because particles from the fingerprint inevitably adhere to the ATR crystal. Ricci et al, however, contend that ATR imaging gives sufficient spatial resolution to obviate the need for synchrotron infrared, and that the gel lifter technique they employed with ATR imaging is non-destructive [10]. An interesting aspect of the article by Grant et al was the idea of considering a spectral image as a "library" of spectra to be searched against one reference spectrum 
(or a small number of reference spectra). This kind of "reverse searching" to find specific exogenous materials in a fingerprint was demonstrated using a commercial library search engine and a reference spectrum from a commercial infrared library. There are, however, some important differences between standard library searches and the reverse searching of spectral images of fingerprints. This is because the latter involves using a single high quality spectrum to search a "library" consisting of large numbers of "empty" spectra, and small numbers of possibly poor quality target spectra that have sloping baselines and other distortions due to the shape and optical properties of the contaminant particles. Thus it is important to ensure that the search algorithm(s) used are well-suited to this application and/or that the data is pre-processed in an appropriate way.

Summaries of infrared spectral searching algorithms and practices can be found in the literature and online [30-35]. Full spectrum algorithms (as opposed to peak search algorithms, for example) generally compare two spectra with some type of distance measure. This is done by representing each spectrum as either a point or a vector in an $n$-dimensional space, where $n$ is the number of resolution elements (wavelengths) in a spectrum. The most successful and hence most popular algorithms used for spectral comparison (searching and classification) in vibrational spectroscopy are variations on the Euclidean distance (ED), Pearson's correlation coefficient and the vector dot product. First and even second derivative spectra are often used to improve searching success, as differentiation tends to remove the effects of curving baselines and resolve closely spaced peaks. Other spectroscopic application areas, especially remote sensing, have developed their own variations on these algorithms, for use with visible light and NIR reflectance spectral images of landscapes. In remote sensing, the most popular spectral comparison algorithm has been the spectral angle mapper (SAM) [36], which is calculated as a vector-normalized dot-product that gives the cosine of the angle between two vectors (spectra). Although the different types of spectroscopy impose their own intrinsic requirements on search algorithms, there are areas where crossfertilization of ideas can occur. However, differing terminologies and the close inter-relationship of many of the full spectrum search algorithms often leads to confusion.

Table 1 gives some of the common full spectrum search algorithms, and helps to illustrate some of the similarities and inter-relationships. As an example, for reasons of vector geometry, the ED will give the same search ranking as the SAM if the spectra are vector-normalized prior to ED searching. It can also be seen from the formulas that the correlation is essentially the same as the SAM, except that the spectra are centred on their respective mean intensities.

Many variations that attempt to improve on the SAM have been devised for remote sensing applications. An example of these is the spectral similarity scale (SSS) [37], which is a combination of 
the Euclidean distance and correlation measures. In infrared spectroscopy, as noted by Loudermilk et al [34], there has been little change in spectral library searching practice since the 1980s. Brown and Donahue have used Fourier compression and the dot product measure to search a UV-visible library [38], and Lo and Brown have devised methods for searching libraries for components in spectra of mixtures, using Fourier compression and principal components regression $[39,40]$.

Notable recent approaches include the Spectral Code Index (a method for indexing infrared spectral for SQL database searching) [41], and the group voting scheme, which uses complementary information from multiple standard search algorithms [34]. Vandenabeele et al reduced a Raman spectral library of synthetic pigments using principal components analysis (PCA) and carried out Euclidean distance searching using the scores for the first 28 principal components [42]. Neural networks have also been trialled in spectral comparison work [43-45]. Turner et al [46] have developed the spectral identity mapper (SIM) and tested it on infrared spectral image data. This method for spectral comparison improves on the SAM by dealing with the possibility that two or more spectra (vectors) may have the same spectral angle from a reference, yet represent different substances (indeed, an infinite number of vectors lying on a cone around the reference vector can share the same angle to the reference). Meanwhile, some authors in both remote sensing and infrared histopathology have started to use probabilistic measures of similarity between spectra [47] or groups of spectral metrics [48]. Such a measure, known as spectral information divergence (SID), has been shown to have advantages over the SAM, ED and correlation measures $[49,50]$.

\section{Experimental}

To prepare fingerprint samples, a clean (washed with soapy water, rinsed and dried) index finger was rubbed over the face/neck and through the hair to ensure sufficient sebaceous secretions were present. This finger was then lightly pressed into a small amountfew milligrams of the powdered substance of interest. Any excess substance was brushed away with the other hand leaving behind nil or minimal visible (to the naked eye) trace of the substance on the finger. The finger containing the substance of interest was then lightly pressed onto either a metal oxide-coated glass slide (Kevley Technologies) (for reflection experiments) or a silicon window (for transmission experiments).

All samples were analysed using a Digilab/Varian Stingray infrared imaging system, which consisted of an FTS7000 FTIR spectrometer coupled to a UMA600 infrared microscope with a Lancer $64 \times 64$ pixel focal plane array (FPA) detector. Samples on the infrared-reflective slides were analysed in reflection mode, while those on the silicon windows were analysed in transmission mode. All images were collected in the expanded field of view, where each 4096-pixel image tile is around 700 
$\times 700 \mu \mathrm{m}$ in size. Full fingerprint images consisted of $256(16 \times 16)$ tiles, giving an overall area of $1.12 \times 1.12 \mathrm{~cm}$. Groups of $16(4 \times 4)$ pixels were aggregated together to give single pixels so that the overall image contained 65,536 $(256 \times 256)$ spectra. Spectral resolution was set at $16 \mathrm{~cm}^{-1}$, and 16 co-added scans were collected, over the range $4000-900 \mathrm{~cm}^{-1}$ (with the lower end of this range corresponding to the low-energy cut-off for the detector). Using these parameters, images were collected in 1 hour and $20 \mathrm{~min}$, and were spectrally ratioed to a background image collected from a clean slide/window.

Testing and comparison of searching algorithms was carried out on a specially constructed dataset, based on caffeine as a model fingerprint contaminant. To collect representative and realistic spectra of caffeine in this role, pure caffeine powder was deposited scattered sparsely (so that particles did not overlap) onto a silicon window and a single image tile (4096 spectra) was collected. From the spectra in this image, 42 caffeine spectra were selected. This selection process involved manually choosing spectra from the image, followed by searching each spectrum selected against a collection of commercial spectral libraries containing organic compounds and forensic samples. Different grades of spectra were chosen for further use. A large number (31) of the spectra gave caffeine as their closest library match. To make the dataset more realistic, the rest of the spectra were of lower quality, but were still confirmed to be caffeine through a combination of library searching and visual inspection. These 42 spectra were used to augment the 4096 spectra from a single image tile of a non-contaminated latent fingerprint (also collected on a silicon window) to give a 4138-spectrum dataset composed of known caffeine spectra and spectra of pixels that were either empty or contained latent fingerprint deposits.

In the first instance, all images and spectra obtained were viewed using the Digilab/Varian Win-IR Pro 3.4 software. In general, images and/or selected reference spectra were exported to ENVI 4.2 (ITT Visual Information Solutions), where spectral derivatives were calculated when required, and where the image spectra were converted to ASCII text files for importing into Microsoft Excel 2007 for the implementation and comparison of the different spectral search algorithms. For principal component (PCA) searching, spectra were exported from Excel in comma separated variable (CSV) format into Pirouette 3.11 (Infometrix). The PCA results from Pirouette were exported directly back into Excel.

As mentioned above, Microsoft Excel 2007 was generally used to implement and compare the various search algorithms. Since the desired outcome of a search of the 4138-spectrum dataset against caffeine was to have the 42 confirmed caffeine spectra fill the first 42 "hits" (when the spectra were ranked according to similarity to the reference spectrum), the spreadsheet rows 
representing the caffeine spectra were colored according to spectral quality for easy assessment of algorithm performance.

Large $(65,536$-spectrum) fingerprintimages of fingerprints contaminated with pharmaceuticals or explosives residues were exported into ENVI for derivatization and classification/searching using the SAM.

\section{Results and discussion}

Preliminary trials (not detailed above) on reverse library searching were conducted using commercial infrared library search software. In these trials, micro-reflectance spectra from images of contaminated fingerprints were converted into a library that was then searched against a high quality reference spectrum of a contaminant (a pharmaceutical, a drug of abuse, or an explosive), using the three algorithms available (nominally, Euclidean distance, first derivative Euclidean distance and correlation). The search results were sorted by hit quality index (HQI) from closest to poorest matches, and attempts were made to devise a method for determining a threshold HQI above which a positive result could be assured, and below which only non-matching ("empty") spectra would be found. Once a threshold had been determined, using an abrupt change in the HQI, the location of "matching" spectra could be mapped back onto the fingerprint image. The chief drawback of this approach was the inability to vary the search algorithm beyond the three available, all of which gave false positives (apparently "empty" spectra which had higher HQI values than many genuine contaminant spectra) and false negatives (confirmed spectra of the contaminant which had lower HQI than many "empty" spectra). Another problem was the spectral distortion (anomalous dispersion) which afflicts many reflectance spectra: although the image spectra were collected from infrared-reflective slides (which can yield high quality transmission-reflection spectra of fine particles in ideal cases), in practice, the random size, shape and surface quality of the particles meant that the contaminant spectra suffered from varying levels of distortion, making the comparison of these spectra with a single reference spectrum very difficult on occasion. Finally, it could be postulated that the algorithms as implemented in the commercial software would not be optimised for the task of "reverse" library searching as described in the Introduction, where the library contains a high number of poor quality spectra. However, despite these issues, some fingerprint images were very successfully searched using the commercial software, and results for cocaine and PETN (pentaerythritol tetranitrate) are shown in Figures 1 and 2. In one experiment, the FTIR image of a fingerprint contaminated with paracetamol was searched for that substance. As most of the paracetamol had been brushed away prior to the deposition of the fingerprint, only three discrete spectra in the image (out of 65,536 ) could be identified as paracetamol. This demonstrated the 
power of spectral image searching, and also enabled the detection limit of this method to be estimated. Given that the pixels in this case were $44 \mu \mathrm{m}$ across, and assuming a spherical particle of about $22 \mu \mathrm{m}$ radius at one of these three pixels, we can estimate a mass of $0.06 \mu \mathrm{g}$ as the detection limit for paracetamol.

Further work was conducted with three major changes in approach: (i) algorithms were implemented in a spreadsheet, giving complete control and knowledge of the processing and comparison of spectra; (ii) spectral images were collected in transmission mode from fingerprints deposited on silicon windows; (iii) in order to assess the relative abilities of different algorithms, the 4096 spectra from a latent (uncontaminated) fingerprint image were augmented with 42 confirmed contaminant (caffeine) spectra chosen as described in the Experimental section. This meant that in comparing the success of the different algorithms, there would be no ambiguity as to the identity of any spectra, but all spectra would still have been collected under identical conditions through the imaging of a fingerprint.

The performance of the following algorithms (SAM, ED, correlation, Manhattan distance, PCA-ED, SSS, SIM) was compared as described above for three different forms of the spectral data: undifferentiated (but range scaled or "normalized" over a range of 0 to 1), first derivative, and second derivative. The differentiated spectra were not range scaled. The results for these three sets of experiments are summarized below:

Undifferentiated spectra

It was initially expected that range scaling would allow the algorithms to achieve their best results with undifferentiated data. To reiterate, the best outcome for an algorithm (in a search against a caffeine reference spectrum) was for it to rank the 42 caffeine spectra above all of the other 4096 spectra in the comparison dataset. With the data in this form, none of the algorithms performed well. The Euclidean distance, for example, ranked the caffeine spectra randomly between $328^{\text {th }}$ and $4088^{\text {th }}$. The SAM method did rank caffeine spectra as the three most similar to the reference, but the rest were randomly ranked down to $3252^{\text {nd }}$. The correlation algorithm performed slightly better than the SAM, with 7 caffeine spectra ranked in the top ten hits, but gave random rankings following these. All other methods performed more poorly than this, and so in comparison, although correlation and SAM performed somewhat better than the rest, none of the algorithms could reliably distinguish fingerprint deposit spectra from those of caffeine. The PCA-ED method performed particularly poorly. When the principal components were examined after PCA was run on the image and reference spectra, it was obvious that the major components contained mostly 
noise and baseline artifacts, due to the poor quality of many of the spectra, and so this method seemed particularly unsuited to the task.

When the caffeine spectra in the dataset were examined, it became obvious that this is not a typical spectral searching operation, because of the poor quality of many of these spectra. High noise levels and severe baseline curvature were common problems. The scaling process was actually inappropriate for these spectra, as often it was the baseline itself that was expanded over the full intensity range. However, while the unscaled spectra gave better results, the algorithms still performed poorly. Rather than investigate baseline correction algorithms, it was decided to study the effects of differentiation on the searching performance of the algorithms.

First derivative spectra

Of the 42 caffeine spectra in the dataset, 31 were identified visually as being of better quality than the other 11. Once the spectral dataset had been differentiated and ranked according to Euclidean distance from the reference, most (29) of these better quality caffeine were ranked at the top of the list. However, several fingerprint spectra were ranked before caffeine spectra, and the two lowest ranked caffeine spectra (out of the 42 ) were found at the $44^{\text {th }}$ and $45^{\text {th }}$ positions. Using SAM, this situation improved: 36 of the 42 caffeine spectra were ranked at the top of the list, but five fingerprint spectra were ranked higher than the lowest-ranked caffeine spectrum, which was at $50^{\text {th }}$. The correlation algorithm performed identically to the SAM in its ranking of the caffeine spectra, with small differences in order occurring further down the hit list. The performance of the SSS was slightly poorer than that of the SAM, with 32 out of the 42 caffeine spectra appearing at the top of the list, and the remainder of the list appearing similar to that generated by the SAM. The other methods performed poorly using this data, and it was clear that they are inferior for this type of searching. An overall comparison shows that the SAM and the correlation methods gave the best results, followed by the ED and then the SSS, which is the combination of the ED and correlation methods.

\section{Second derivative spectra}

Searching using second derivative spectra gave the best results for most of the search algorithms. Now, all three of the ED, SAM and correlation methods listed all of the caffeine spectra at the top of their hit lists, albeit in slightly different orders. The SSS performed very well, but not quite as well as with the first derivative, and not quite as well as the three methods above (four caffeine spectra ranked lower than some fingerprint spectra). PCA searching put 21 caffeine spectra at the top of the list, but the remaining spectra were interspersed with fingerprint spectra in the hit list, and one was 
ranked $4038^{\text {th }}$. Here for the first time, the SIM was tested, and it performed very well: all but four caffeine spectra were at the top of the list; these four were ranked down to $70^{\text {th }}$. When comparing the ED, SAM and correlation results to each other, the only distinction that could be made was that the ED did not order the caffeine spectra as closely to what a visual inspection of the spectra might have suggested. This observation is based on the relative rankings of some of the highest and lowest quality caffeine spectra. On the other hand, the SAM and correlation results were very similar, with only minor differences in the rankings of the known caffeine spectra.

Truncation of spectra

In an attempt to improve searching results and to reduce computation time, some algorithms (ED, SAM, correlation and SSS) were tested on truncated versions of the second derivative dataset discussed above. When the data was truncated slightly (spectral range reduced to $987-3526 \mathrm{~cm}^{-1}$ to eliminate noisy data at low and high wavenumbers), the four methods performed equally well, with the ED improving upon its performance with the full dataset. On the other hand, when the data was truncated to the "fingerprint" region of the spectrum (987-2005 $\mathrm{cm}^{-1}$ in this case), the ED and SSS gave poorer results, while the SAM performance stayed high. For this reason, and since the SAM could be implemented with great ease and functionality in the ENVI software, it was decided that further tests would almost exclusively use this algorithm on truncated second derivative spectra.

Further testing

In this experiment, a 65,536 $(256 \times 256)$-spectrum image of a fingerprint contaminated with three powdered pharmaceuticals (caffeine, aspirin and diazepam) was collected from a silicon window. The aim here was to observe the performance of the best search algorithms (SAM and ED on second derivative spectra) in searching a dataset when three different exogenous substances were present. For example, would all three substances be correctly identified? Would a search for (say) caffeine rank diazepam and aspirin spectra higher than empty or fingerprint spectra? SAM and ED second derivative searches for each of the three substances (against a high quality reference spectrum in each case) were performed. Figure 3 shows the results of the searches performed on this data. In the caffeine search, only one spectrum was positively identified as caffeine by the SAM (the first spectrum in the "hit list"), although this spectrum did not receive a high ranking by ED. It appeared that most of the caffeine particles had been wiped away during the removal of excess material on the finger. SAM searches for diazepam and aspirin were also successful, with mostly unambiguous results. Aspirin proved to be far more persistent than the other two substances, as its spectra were by far more numerous. During a visual examination of the SAM searching results, a spectrum which 
might represent a mixture of aspirin and diazepam was found, probably a result of a pixel being occupied by particles of both substances. This spectrum was ranked $23^{\text {rd }}$ in both the aspirin and diazepam searches. This type of result reinforces the standard maxim of spectral searching, that the operator must visually confirm search results. Examining the results of the three searches in more detail, it was observed that in any given search, the spectra of the two non-target contaminants usually ranked very low, but (as might be expected) were clustered together with like spectra.

Another test for the SAM was to observe the result when fingerprint and contaminant spectra were searched against a reference spectrum of a substance which was not present. In this case, the image containing aspirin, caffeine and diazepam described above was searched for paracetamol. Once the hit list of the 65, 536 spectra was inspected, it was determined that none of the previously identified possible aspirin, caffeine or diazepam spectra appeared at the top of the list, and most of these were ranked lower than 10,000 . In situations like these, it is instructive to observe the value of the spectral angle (and its rate of change with respect to hit number) at thresholds between the contaminant and "empty" spectra.

Searching for illicit substances in fingerprints

As mentioned above, the SAM is implemented in the ENVI software package. In this software, it can be used to indicate (using a single color) pixels in a spectral image whose spectra fall within a threshold angle to a reference spectrum. Alternatively, it can assign a color intensity based on the magnitude of the spectral angle. The latter functionality was used to examine fingerprints contaminated with explosives. In these tests, FTIR images of three fingerprints were collected from silicon windows; these were contaminated with ammonium nitrate/fuel oil, Det cord and Pentex booster respectively. Ammonium nitrate was successfully found in the first image in high quantities, so high that it was possible to form a high contrast image of the fingerprint using the intensity of ammonium nitrate spectral peaks from the raw spectra. Using a reference spectrum of PETN (a component of Det cord collected in the laboratory), PETN particles were identified at several pixels in the second image, but it was difficult to form an image of the entire fingerprint at any frequency due to a lack of material deposited and because of possible smearing of the print. PETN is also a component of the Pentex booster, but was not found in the third fingerprint when searched. No image of this fingerprint could be formed at any frequency. In this case, it seemed that the donor's finger was too clean, and lacking in secretions which might have caused PETN to adhere, and which might also have yielded an image of the fingerprint ridges when deposited. Skin particles were detected, and although TNT may have been present, a search for it was not possible due to a lack of reference spectra or material. 


\section{Conclusion}

Spectral or chemical imaging yields large numbers of spectra that can be used to form chemically specific images of a sample, through either univariate or multivariate representations of the data. However, in many applications, spectral imaging is more useful as a means for collecting thousands of spectra simultaneously across a sample area. In forensic science, this means a wealth of spatially resolved information about trace evidence that may be present; this information can usually be collected quickly and non-destructively. Spectral searching of chemical images suggests itself quite readily [7reffrant et al] as a variation on traditional spectral library searching, with the distinction that large numbers of the spectra in the "library" may be devoid of useful information, while those spectra that are of interest may be of poor quality, since the operator does not have the luxury of being able to prepare an ideal sample. These aspects of searching for (say) trace evidence on a surface mean that not all of the commonly used library searching algorithms may be appropriate to the task.

In the current study, the spectral angle mapper (SAM, or vector normalized dot product measure; also called cosine correlation analysis), along with the (Pearson's) correlation measure, when used on second derivative spectra, were found to give the best results when searching for exogenous substances among the spectra in an FTIR image of a latent fingerprint. Other methods, such as the Euclidean distance and the spectral identity mapper, performed almost as well as the SAM, particularly for carefully truncated second derivative data. However, the SAM was selected here for further testing as it is easier to implement and its use is well developed in a commercial software package intended mainly for remote sensing scientists. The success of SAM and the related correlation measure comes as no surprise considering that correlation on second derivative spectra is a popular spectral distance calculation method used prior to hierarchical cluster analysis in infrared histopathology [51]. In that case, the application of spectral derivatives is typically carried out with smoothing to remove some of the noise created in the differentiation process. What is, perhaps, surprising is that the spectral comparison methods designed to improve upon the SAM (such as the spectral similarity scale or the spectral identity mapper) did not perform better than the SAM with the data used for testing here. The SSS is designed for remote sensing data [37], but the SIM was originally tested by Turner et al [46] using infrared spectra. The most likely explanation is that, because these methods performed very well, comparisons using more data are required before a final verdict can be given. Our own attempts to improve on SAM using combination measures similar to the SSS failed to improve on the SAM (or correlation), usually because they were based on 
the SAM or correlation and another measure which did not perform as well as these and hence dragged the overall performance down.

The conclusions reached here would probably apply to Raman spectral images, but the desirability of automated searching of spectral images is not limited to vibrational spectroscopy, and could, for example, be applied in the forensic analysis of visible light spectral image data of gunshot residue. However, other modes of spectroscopy would require their own assessment of algorithms and searching practices: for example, the use of derivatives in UV-visible spectroscopy would see a loss of useful information, as the typically broad spectral features would be levelled out, and the spectra would become significantly noisier.

Insofar as the searching of infrared images of fingerprints is concerned, practical sampling considerations suggest that the situations where this technique may be useful are those where (i) a suspect in custody is directed to deposit prints onto an ideal surface (such as a silicon window or an ATR crystal); (ii) where fingerprints have been deposited into small, flat metallic objects and (iii) more importantly, where tape- or gel-lifted fingerprints can be transferred onto an ideal surface. The ability to generate the best possible spectral quality by using transmission or ATR imaging will help determine the possible success of subsequent spectral searching. Other techniques are becoming available for the detection of exogenous materials in fingerprints: these include new mass spectrometry (MS) methods $[52,53]$ and methods which involve the fluorescent tagging of specific target compounds within a fingerprint using antibodies attached to magnetic particles [54-56]. However, the MS methods will be destructive, and the tagging methods will require different taggant particles to be prepared for each target compound (and thus assumptions about which target molecules might be present). Therefore it seems that spectral searching will have an important role to play because of its non-destructive nature and relative ease of implementation once spectral imaging equipment becomes more widely accessible and searching protocols have been established for different spectroscopic modes/regions. However, further testing of more recent innovations in spectral similarity measures (such as SID) is required.

\section{Acknowledgements}

We would like to thank the Australian Federal Police for the donation of some of the contaminated fingerprints analysed in this work. 


\section{References}

1. $\quad$ Mercado AG, Janni J, Gilbert B (1995) Proc SPIE-Int Soc Opt Eng 2511: 142-152

2. Bartick E, Schwartz R, Bhargava R, Schaeberle M, Fernandez D, Levin I (2002) Spectrochemical analysis and hyperspectral imaging of latent fingerprints. Abstracts, 16th Meeting of the International Association of Forensic Sciences, Montpellier, France, pp. 61-64.

3. Exline DL, Wallace C, Roux C, Lennard C, Nelson MP, Treado PJ (2003) J Forensic Sci 48: 10471053

4. Bartick EG, Williams DK, Peters HL, Schwartz RL, Crane NJ, Bhargava R, Fernandez D, Huffman SW, Levin I (2005) Spectrochemical analysis and spectral imaging of latent fingerprints and trace evidence included within the prints. Abstracts, 37th Middle Atlantic Regional Meeting of the American Chemical Society, New Brunswick, NJ, United States.

5. Tahtouh M, Kalman JR, Roux C, Lennard C, Reedy BJ (2005) J Forensic Sci 50: 64-72

6. Miskelly GM, Wagner JH (2005) Forensic Sci Int 155: 112-118

7. Grant A, Wilkinson TJ, Holman DR, Martin MC (2005) Appl Spectrosc 59: 1182-1187

8. Ricci C, Chan KLA, Kazarian SG (2006) Appl Spectrosc 60: 1013-1021

9. Crane NJ, Bartick EG, Perlman RS, Huffman S (2007) J Forensic Sci 52: 48-53

10. Ricci C, Bleay S, Kazarian SG (2007) Anal Chem 79: 5771-5776

11. Ricci C, Phiriyavityopas P, Curum N, Chan KLA, Jickells S, Kazarian SG (2007) Appl Spectrosc 61: 514-522

12. Tahtouh M, Despland P, Shimmon R, Kalman JR, Reedy BJ (2007) J Forensic Sci 52: 1089-1096

13. Payne G, Wallace C, Reedy B, Lennard C, Schuler R, Exline D, Roux C (2005) Talanta 67: 334344

14. Flynn K, O'Leary R, Lennard C, Roux C, Reedy BJ (2005) J Forensic Sci 50: 832-841

15. Flynn K, O'Leary R, Roux C, Reedy BJ (2006) J Forensic Sci 51: 586-596

16. Chan KLA, Kazarian SG (2006) Analyst 131: 126-131

17. Champod C, Lennard C, Margot P, Stoilovic M (2004) Fingerprints and other ridge skin impressions. CRC Press, Boca Raton, FL

18. Ramotowski R (2001) In: Lee HC, Gaensslen RE (eds) Advances in fingerprint technology. 2nd edn. CRC Press, Boca Raton, FL

19. Wilkinson TJ, Martin MC, McKinney WR, Perry DL (2004) Application of FTIR spectromicroscopy to the analysis of latent human fingerprints. 227th National Meeting of the American Chemical Society. Abstracts of Papers - ACS, Anaheim, CA, pp. Paper IEC-157.

20. Wilkinson TJ, Perry DL, Martin MC, McKinney WR, Thompson AC (2001) Applications of synchrotron technology to the study of latent human fingerprints. 222nd National Meeting of the American Chemical Society. Abstracts of Papers - ACS, Chicago, IL, pp. Paper IEC-036.

21. Williams DK, Schwartz RL, Bartick EG (2004) Appl Spectrosc 58: 313-316

22. Day JS, Edwards HGM, Dobrowski SA, Voice AM (2004) Spectrochimica Acta 60A: 563-568

23. Hemmila A, McGill J, Ritter D (2008) J Forensic Sci 53: 369-376

24. Cheng C, Kirkbride TE, Batchelder DN, Lacey RJ, Sheldon TG (1995) J Forensic Sci 40: 31-37

25. Hayward IP, Kirkbride TE, Batchelder DN, Lacey RJ (1995) J Forensic Sci 40: 883-884

26. Day JS, Edwards HGM, Dobrowski SA, Voice AM (2004) Spectrochim Acta 60A: 1725-1730

27. Edwards HGM, Day JS (2006) Vib Spectrosc 41: 155-159

28. West MJ, Went MJ (2008) Forensic Sci Int 174: 1-5

29. West MJ, Went MJ (2009) Spectrochim Acta 71A: 1984-1988

30. Lowry SR, Dalrymple DM, Rosenthal RJ (1988) Mikrochim Acta 1: 89-92

31. Lowry SR (2002) In: Chalmers JM, Griffiths PR (eds) Handbook of vibrational spectroscopy. Wiley, New York

32. Griffiths PR, De Haseth JA (2007) Fourier transform infrared spectrometry. 2nd edn. WileyInterscience, Hoboken, N.J.

33. Günzler H, Gremlich H-U (2002) IR spectroscopy : an introduction. Wiley-VCH, Weinheim ; Cambridge 
34. Loudermilk JB, Himmelsbach DS, Barton FE, II, de Haseth JA (2008) Appl Spectrosc 62: 661-670

35. Algorithms used in spectroscopy (2009) Thermo Fisher Scientific Inc. http://www.thermo.com/com/cda/resources/resources detail/1,13229,00.html. Accessed 1 Feb 2009

36. Kruse FA, Lefkoff AB, Boardman JW, Heidebrecht KB, Shapiro AT, Barloon PJ, Goetz AFH (1993) Remote Sens Environ 44: 145-163

37. Sweet JN (2004) The spectral similarity scale and its application to the classification of hyperspectral remote sensing data. 2003 IEEE Workshop on Advances in Techniques for Analysis of Remotely Sensed Data, IEEE, pp. 92-99.

38. Brown CW, Donahue SM (1988) Appl Spectrosc 42: 347-352

39. Lo SC, Brown CW (1991) Appl Spectrosc 45: 1628-1632

40. Lo SC, Brown CW (1991) Appl Spectrosc 45: 1621-1627

41. Li JF, Fan BT, Doucet J-P, Panaye A (2003) Appl Spectrosc 57: 858-867

42. Vandenabeele P, Hardy A, Edwards HGM, Moens L (2001) Appl Spectrosc 55: 525-533

43. Luinge HJ, Van der Maas JH, Visser T (1992) Proc SPIE-Int Soc Opt Eng 1575: 499-500

44. Mittermayr CR, Drouen ACJH, Otto M, Grasserbauer M (1994) Anal Chim Acta 294: 227-242

45. Yang H, Griffiths PR (1999) Anal Chem 71: 751-761

46. Turner JF, II, Zhang J, O'Connor A (2004) Appl Spectrosc 58: 1308-1317

47. Chang C-I (2000) IEEE transactions on information theory 46: 1927-1932

48. Fernandez DC, Bhargava R, Hewitt SM, Levin IW (2005) Nat Biotechnol 23: 469-474

49. Chang C-I (2003) Hyperspectral imaging : techniques for spectral detection and classification. Kluwer Academic/Plenum Publishers, New York

50. van der Meer F (2006) Int J Appl Earth Obs Geoinf 8: 3-17

51. Diem M, Romeo M, Boydston-White S, Miljkovic M, Matthaeus C (2004) Analyst 129: 880-885

52. Szynkowska MI, Czerski K, Rogowski J, Paryjczak T, Parczewski A (2009) Forensic Sci Int 184: e24-e26

53. Ifa DR, Manicke NE, Dill AL, Cooks RG (2008) Science 321: 805

54. Hazarika P, Jickells SM, Russell DA (2008) Analyst 134: 93-96

55. Hazarika P, Jickells SM, Wolff K, Russell DA (2008) Angew Chem, Int Ed 47: 10167-10170

56. Leggett R, Lee-Smith EE, Jickells SM, Russell DA (2007) Angew Chem, Int Ed 46: 4100-4103 
Table 1

Some of the more popular spectral comparison algorithms. The spectrum represented by $x_{i}(i=1$, $2, \ldots, n)$ is being compared to the reference $r_{i}$. The term $x_{i}$ is the intensity at the ith frequency position, $r_{i}$ is the corresponding value from the reference spectrum, and $n$ is the number of frequency resolution elements. For most of these measures, as given, a smaller value indicates greater similarity. For the correlation measure, a smaller value of $1-\rho$ indicates greater similarity.

\begin{tabular}{|l|l|}
\hline Euclidean distance & $E D=\left(\sum_{i=1}^{n}\left(x_{i}-r_{i}\right)^{2}\right)^{\frac{1}{2}}$ \\
\hline $\begin{array}{l}\text { Manhattan (city-block) } \\
\text { distance (absolute } \\
\text { differences) }\end{array}$ & $M D=\sum_{i=1}^{n} \mid x_{i}-r_{i} \|$ \\
\hline $\begin{array}{l}\text { Dot product } \\
\text { Spectral angle mapper }\end{array}$ & SAM $=\sum_{i=1}^{n} x_{i} r_{i}$ \\
\hline \begin{tabular}{c}
$\cos ^{-1}\left(\frac{\sum_{i=1}^{n} x_{i} r_{i}}{\left(\sum_{i=1}^{n} x_{i}^{2}\right)^{\frac{1}{2}}\left(\sum_{i=1}^{n} r_{i}^{2}\right)^{\frac{1}{2}}}\right)$ \\
\hline (Pearson's) correlation
\end{tabular} & $\rho=\frac{\sum_{i=1}^{n} x_{i} r_{i}-\sum_{i=1}^{n} x_{i} \sum_{i=1}^{n} r_{i}}{\left(\left[\sum_{i=1}^{n} x_{i}^{2}-\sum_{i=1}^{n}\left(x_{i}\right)^{2}\right]\left[\sum_{i=1}^{n} r_{i}^{2}-\sum_{i=1}^{n}\left(r_{i}\right)^{2}\right]\right)^{\frac{1}{2}}}$ \\
\hline
\end{tabular}



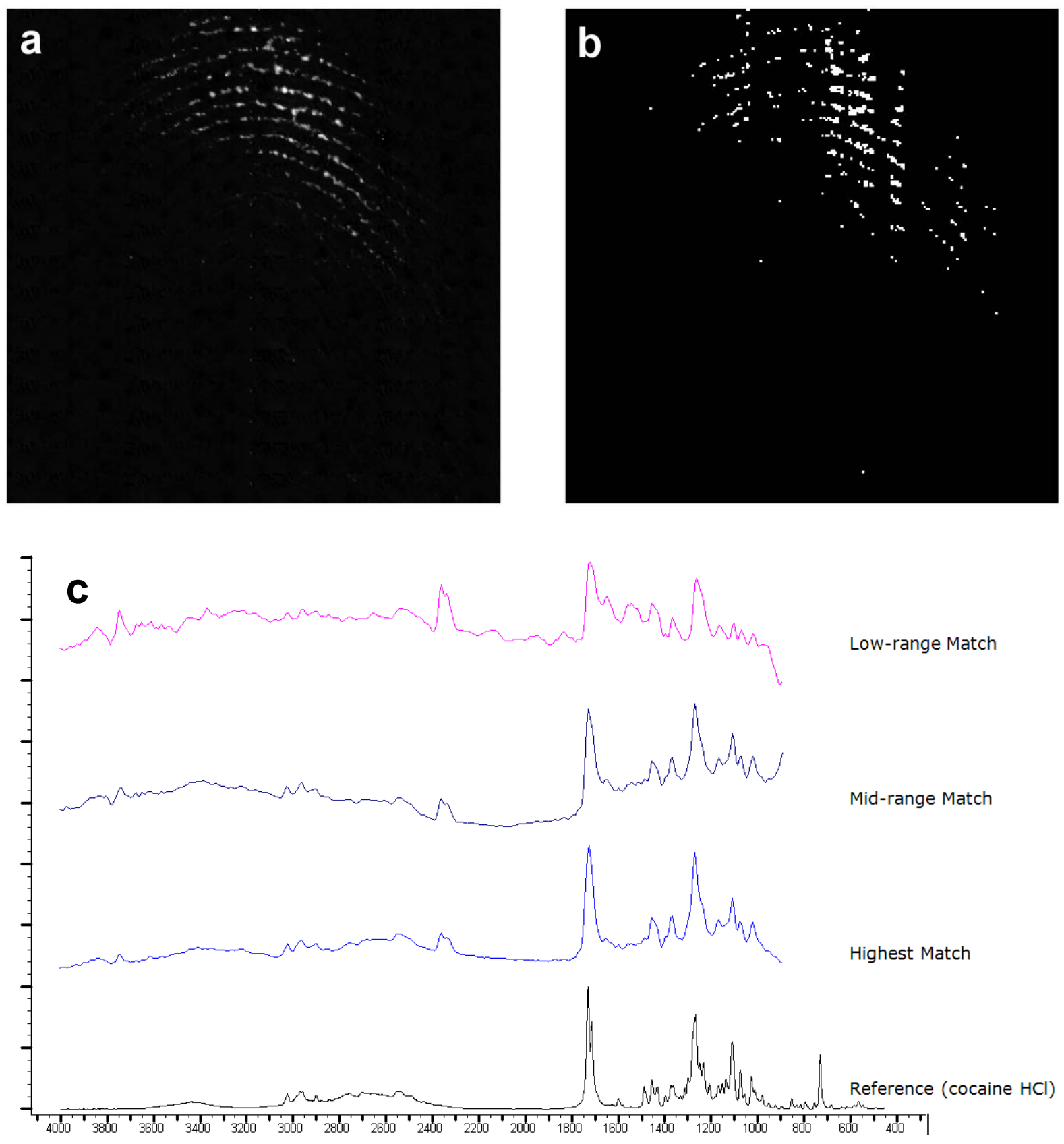

Fig. 1 a Infrared spectral image $\left(256 \times 256\right.$ spectra) of cocaine-contaminated fingerprint at $3 \times x x \mathrm{~cm}^{-1}$. b "Hit map" showing pixels at which cocaine was detected by searching against a cocaine reference spectrum using commercial search software. c Spectra of varying quality detected in the image and the reference spectrum used. 


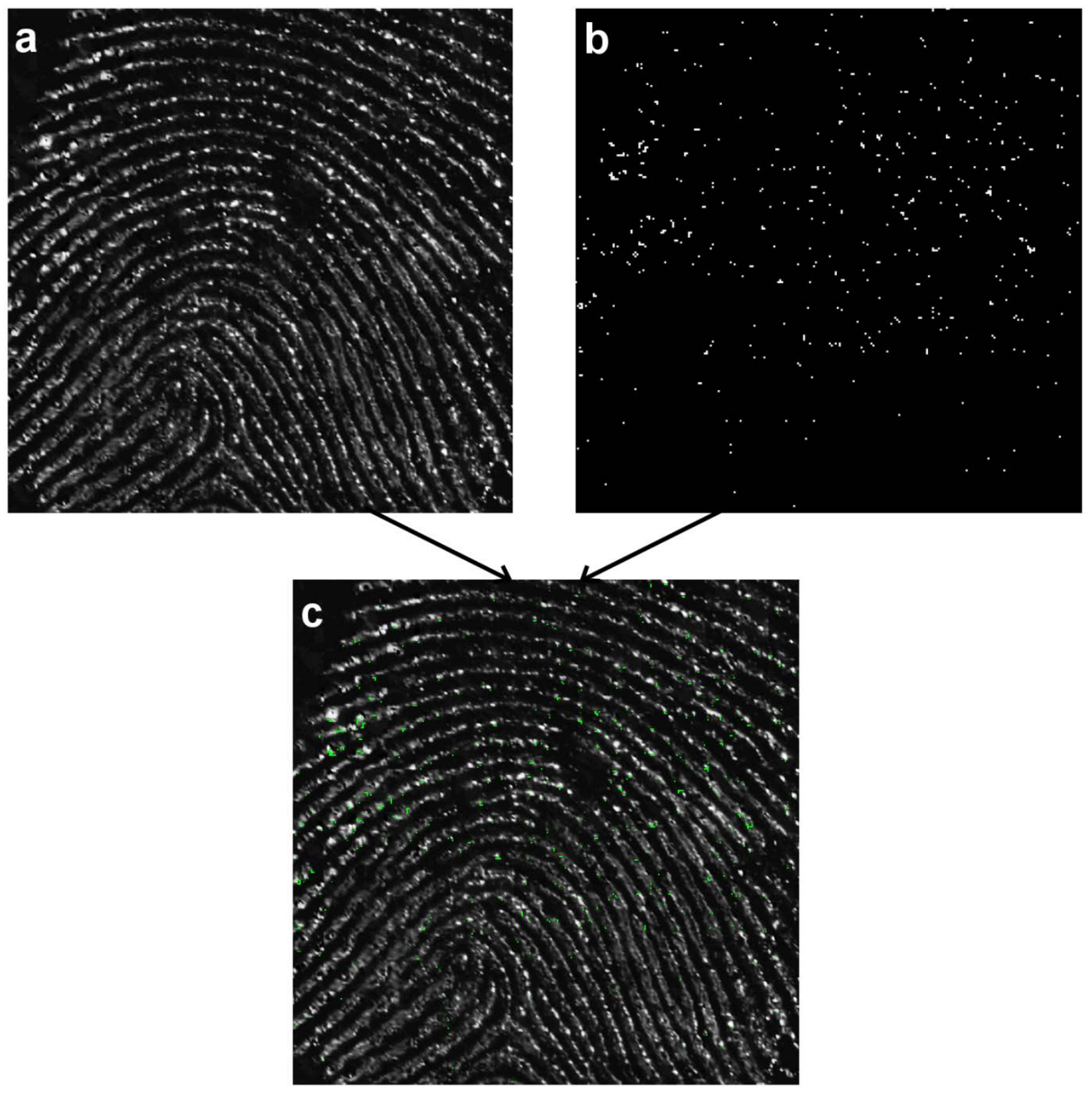

Fig. 2 a Infrared spectral image of PETN-contaminated fingerprint at $2930 \mathrm{~cm}^{-1}$, showing fingerprint deposit only. b "Hit map" showing location of confirmed PETN particles/spectra as found by search against PETN reference using commercial software. c Overlay of PETN hit map (green) on infrared image of fingerprint. 


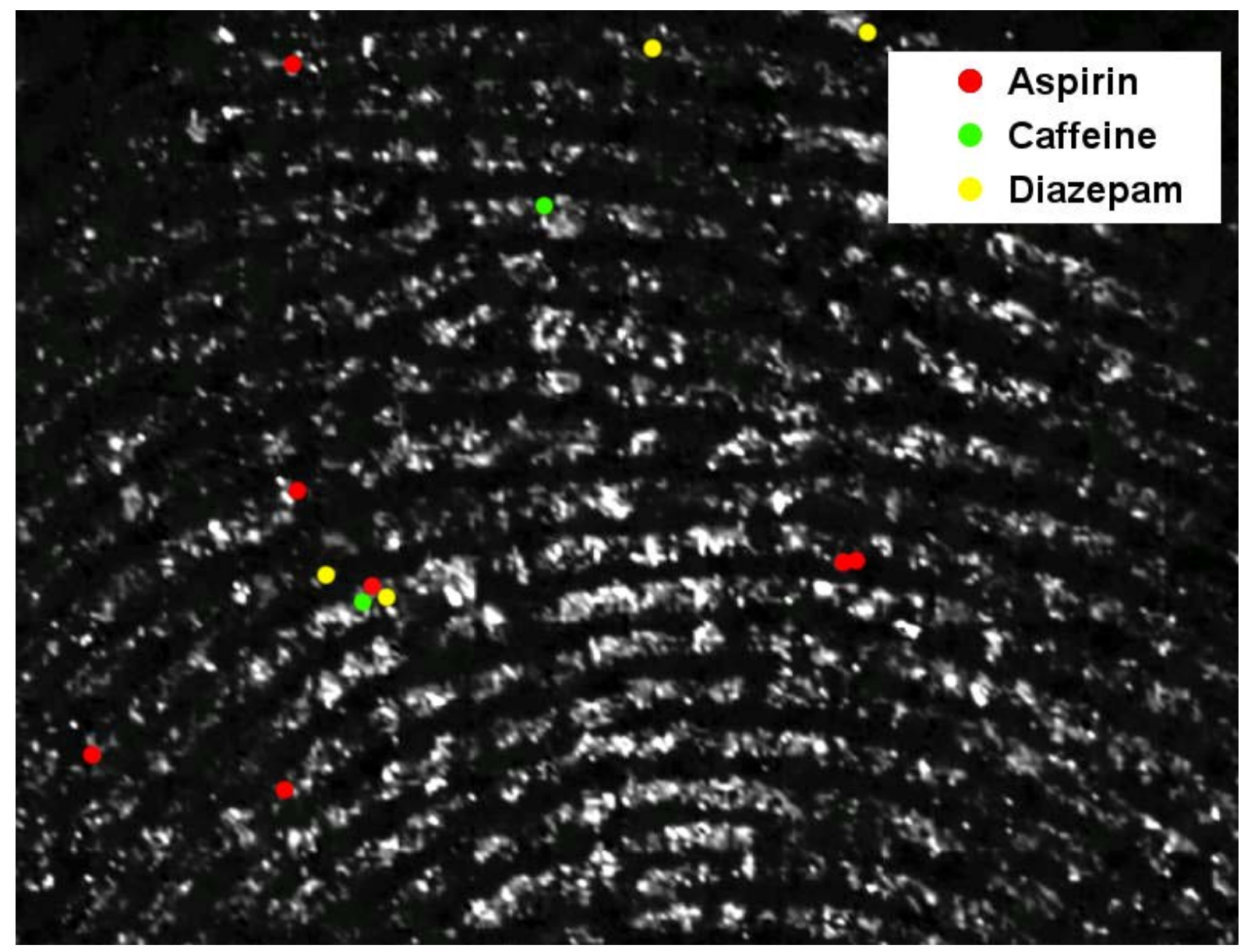

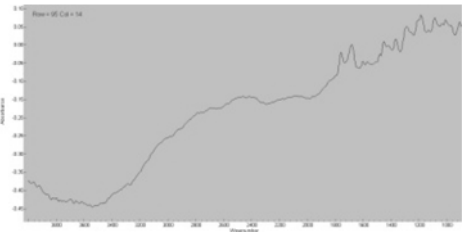

a1) Spectrum most likely to be aspirin

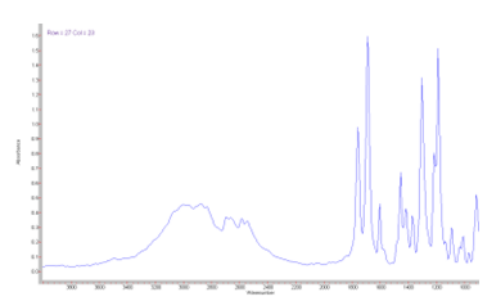

a2) Reference spectrum of aspirin

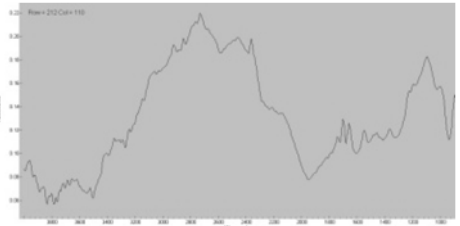

b1) Spectrum most likely to be caffeine

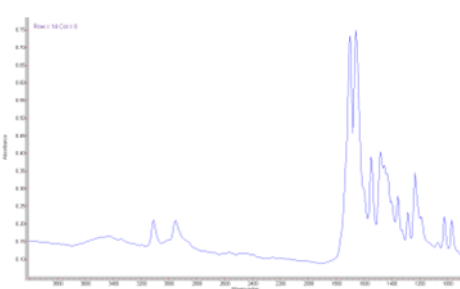

b2) Reference spectrum of caffeine

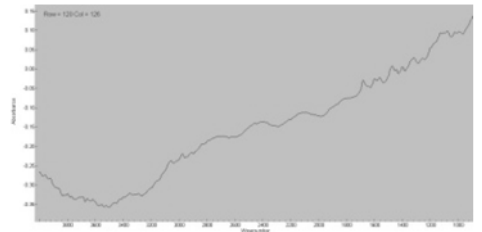

c1) Spectrum most likely to be diazepam

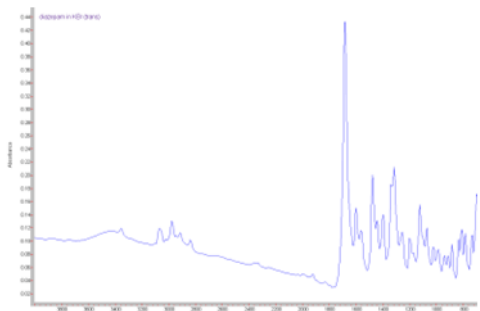

c2) Reference spectrum of diazepam

Fig. 3 Overlay of infrared spectral image of fingerprint at $2930 \mathrm{~cm}^{-1}$ with hit maps for three contaminants, aspirin, caffeine and diazepam, as found by SAM searching of second derivative spectra in image. Highest spectral matches ( $a 1-c 1)$ and reference spectra (a2-b2) for the three compounds are shown underneath. 